\title{
Newborn Screening for Severe Combined Immunodeficiency and T-cell Lymphopenia
}

\author{
Jennifer M. Puck, MD \\ UCSF Department of Pediatrics, Division of Allergy, Immunology and Blood and Marrow \\ Transplantation
}

\section{Summary}

The development of a $\mathrm{T}$ cell receptor excision circle (TREC) assay utilizing dried blood spots made possible universal newborn screening (NBS) for severe combined immunodeficiency (SCID) as a public health measure. Upon being flagged by an abnormal screening test in a SCID screening program, an infant can receive further diagnostic testing for SCID in the neonatal period, prior to onset of infectious complications, to pemit immediate institution of protective measures and definitive, life-saving treatment to establish a functional immune system. SCID screening is now the accepted standard of care in state public health departments across the USA, and it is being adopted in many countries. It has proven effective, with infants having this otherwise inapparent but serious, rare disorder achieving survival and immune reconstitution. In addition to bringing to attention infants with the primary screening target diseases, typical SCID and leaky SCID (due to hypomorphic mutations in known SCID genes), the newborn screening assay for insufficient TRECs in dried blood spots also reveals infants with non-SCID T lymphopenic conditions. Experience has accumulated regarding the range and limitations of diagnoses of newborns with low TRECs and low T cells. Previously unknown immune defects have been discovered, as well as conditions not formerly recognized to have low $\mathrm{T}$ cells in the neonatal period.

\section{Keywords}

hematopoietic cell transplantation (HCT); newborn screening (NBS); primary immunodeficiency (PID); severe combined immunodeficiency (SCID); T cell lymphopenia (TCL); T cell receptor excision circle (TREC)

\section{Introduction}

Severe combined immunodeficiency (SCID) is the term used to describe at least 20 known, and additional unknown, rare genetic disorders in which both $\mathrm{T}$ and $\mathrm{B}$ cell immunity, the components of the adaptive immune system, are lacking. Individuals affected with SCID have absent or very low numbers of $\mathrm{T}$ cells and absent or nonfunctional $\mathrm{B}$ cells, rendering

Address correspondence to: Jennifer M. Puck, MD, UCSF Department of Pediatrics, Box 3118, Division of Allergy, Immunology and Blood and Marrow Transplantation, 555 Mission Bay Blvd South, Rm SC-252K, San Francisco, CA 94143, Phone: 415 502-2090, Jennifer.Puck@ucsf.edu.

Dr. Puck reports that her spouse is employed at Invitae, a gene sequencing company; she has no other financial or personal relationships that could be viewed as presenting a potential conflict of interest. 
them susceptible to life-threatening bacterial, fungal and viral infections with both ordinary and opportunistic pathogens. $(1,2)$ SCID was uniformly fatal in the first year of life prior to 1968, when the first successful treatment was reported in a male infant who received a transplant of bone marrow from his healthy, HLA-matched sibling. (3) Advances in allogeneic hematopoietic cell transplantation (HCT) have been pioneered in SCID since that time, with effective treatment, at least with restoration of $\mathrm{T}$ cell numbers and function, accomplished by HCT from not only matched siblings, but also from alternative donors. Haplo-identical parental bone marrow or mobilized peripheral blood hematopoietic stem cells (PBSC) depleted of T cells have been used, (4) as well as closely matched bone marrow, PBSCs or cord blood from unrelated donors. In addition, enzyme replacement therapy (ERT) is available for adenosine deaminase (ADA) deficient SCID, and autologous hematopoietic cell correction by gene therapy (GT) has been successful for ADA deficient and X-linked SCID, with Artemis-deficient SCID also being tested in a clinical trial. $(1,2,4-$ 7) Best outcomes are achieved if infants are treated early to avoid infectious complications, as documented by single center reports ( 8 ) and by retrospective and prospective multicenter studies from the Immune Deficiency Foundation (9) and Primary Immune Deficiency Treatment Consortium (PIDTC). (5-7)

Because SCID is rare and affected infants appear healthy at birth, and the majority of cases have no recognized positive family history, population-based newborn screening was turned to as a strategy to identify infants with SCID sufficiently early in life to provide treatment before the onset of infectious complications. $(2,8,9)$ Unlike traditional one-on-one diagnostic evaluations of patients, basic immunological research or advancement of new therapies in clinical trials, screening presents a new paradigm for immunological advancement from which unparalleled insights have been gained. However, because screening is imposed upon an entire population with the goal of advancing public health, it is important to appreciate the differences between population-based screening programs vs. clinical care or consented research.

\section{Evolution of the Field of Primary Immunodeficiency}

Figure 1 illustrates my view of the three well established tracks that over the past several decades, with many cross-fertilizations between them, as well as input from other disciplines, have advanced our levels of scientific and medical knowledge of primary immunodeficiency (PID) and improved patient care. The top track is derived from clinical observers, whose interactions with patients suspected to have a rare disorder of host defenses begin with the history, physical examination and interpretation of laboratory tests, microbiologic cultures and imaging. In the past, this has been the sole entry point for individuals affected with PID the into the medical system, and remains so for most cases. The invaluable phenotypic picture that emerges from astute history of exposures and family background, description of syndromic features or failure to thrive, and documentation of unusual organisms causing infections as well as immunologic parameters is essential to further progress. The recent explosion in identification of genetic mutations that underlie immune phenotypes, with over 380 described in the most recent listing by the Inborn Errors of Immunity Committee of the International Union of Immunologic Societies (IUIS) (10) has made immunodeficiency gene diagnosis the next step in this track, followed by assembly 
of the genotype/phenotype spectrum as enough cases with mutations in each particular gene are reported to recognize differences as well as similarities. At this time, gene sequencing, including gene panels, exomes and even whole genomes, is becoming more available, and at the current limit of this clinical approach is the difficulty in interpreting gene variants. The importance of sharing gene variants associated with rare disorders along with phenotype data and evidence for pathogenicity in publicly available databases, such as ClinVar, (11) cannot be overstated, but PIDs are so rare and their mutation spectrum so broad that databased and in silico prediction tools are likely to remain insufficient to establish whether a new variant is pathogenic.

The middle track in the evolution of knowledge concerning PIDs shown in Figure 1 is basic research, in which fundamental discoveries have been based on observations of how the immune system works, usually without reference to a particular patient. Model organisms, particularly the mouse, have provided insight from creation of closely related strains with defined genotypes, in which many animals can be studied in a controlled environment, and researchers can follow responses to infectious or other challenges and study the roles of cells isolated from organs in a manner not possible in humans. Although mouse models do not reliably mimic the phenotypes of all human PIDs with orthologous gene defects, the immunological techniques, biochemistry and biology from basic research have been translated into clinical research laboratories that have continuously increased the sophistication of patient evaluations.

Moreover, understanding of immunologic mechanisms has dramatically shifted the therapeutic lower track (Figure 1) in the field of PID, which represents treatment modalities available to clinicians to prolong life and improve the health of individuals affected with PIDs. In this track, very few options were available for treating patients until recently. For decades after the first recognition that genetic factors could lead to susceptibility to infections or to autoimmune or inflammatory conditions, the only treatments available addressed these complications, rather than the underlying disease processes themselves. Nonetheless, such breakthroughs as antibiotics were lifesaving, for example changing the original "fatal granulomatous disease" (12) due to absence of the respiratory burst leading oxidative intracellular killing of S. aureus and other infections to "chronic granulomatous disease." (13) Similarly, steroids were the primary treatment for autoimmunity, despite unwanted side effects, not the least of which was making already immune deficient patients even more susceptible to infections.

Certain primary immunodeficiencies have been proven amenable to treatments that bypass the genetic defect in a generic manner. These treatments include administration of immunoglobulin $\mathrm{G}(\mathrm{IgG})$ pooled and purified from large numbers of healthy donors, a successful generic measure to circumvent insufficient production of specific antibodies by $\mathrm{B}$ cells. HCT, already mentioned above as life-saving treatment for SCID, can be successful for any disease in which the underlying defect resides within hematopoietic stem cells (HSC), which are the source of all mature blood cell lineages, including T, B and NK lymphocytes, neutrophils and monocytes, platelets and erythrocytes. Engraftment of HSCs, from a healthy, HLA-matched donor, can generate a continuous supply of normally functioning differentiated cells in all blood lineages, effecting durable cures; but positive 
outcomes can also be realized even with mixed chimerism following HCT, such as with restoration of $\mathrm{T}$ cell immunity in SCID, even if $\mathrm{B}$ cell engraftment is not achieved and patients must remain on long-term IgG infusions. $(4,6)$ Because most patients lack a healthy, HLA-matched sibling donor, finding alternative donors for PID patients has been greatly facilitated by the growth of unrelated donor registries and cord blood repositories.

Improvements in transplant protocols and supportive care have been required to manage graft rejection and graft vs. host disease (GVHD), both of which, along with toxic effects of pre-HCT chemotherapy conditioning regimens and infections continue to pose significant risks of morbidity and mortality from this procedure.

Finally, the most recent and exciting treatment modalities, translating the findings of the basic research track into the clinic, are specific therapies targeted to immunologic pathways, abnormally functioning gene products, or the particular mutated genes themselves. Therapeutic small molecules and monoclonal antibodies are now revolutionizing care for many autoimmune diseases, taking the place of steroids because they have fewer systemic side effects. Gene therapy, accomplished by lentiviral addition of a cloned, correct copy of a gene into HSCs of a patient harboring a gene defect, has proven so successful for ADA SCID and X-linked SCID that it is poised to become standard therapy. (14-17) Similar gene addition trials are in progress for other PIDs. Although low doses of chemotherapy appear to be required to make space in bone marrow niches for engraftment of corrected HSC, the difficulties with allogeneic HCT, rejection, and GVHD are avoided. Further advances promise to make possible editing of a patient's own defective gene locus in situ, preserving physiologic gene expression and regulation (18). These gene-based therapies are of course possible only for patients whose specific gene defect is known.

\section{Screening for SCID as a Public Health Measure}

Screening an entire population for primary immunodeficiency is a radical departure from previous scientific and medical paradigms in this this field (Figure 1, lower right). Perhaps the only comparable concept in immunology is screening for HIV, now performed in the developed world for pregnant women giving birth so that newborns who have been exposed can receive effective anti-HIV prophylaxis to avoid becoming infected. Instead of being centered on a patient with signs and symptoms of disease, newborn screening applies a simple assay for a biomarker to every infant in a population of whom the vast majority are not affected. Screening can be justifiably undertaken for rare conditions that are not recognized by standard clinical examination, but are treatable, and for which early diagnosis and treatment are essential to achieve a positive outcome. The screening biomarker has to be extremely sensitive, because missing cases due to false negative results defeats the purpose of detecting every affected individual. Screening tests also must be highly specific to avoid excessive cost and anxiety associated with false positive screen results, but some false positives are considered an acceptable tradeoff to avoid false negatives.

Most screening tests are not designed to establish a diagnosis, but rather to signal the potential for a serious condition for which specific follow-up must be initiated. Therefore, screening should be thought of as an integrated program and not merely a test. The program is directed from a public health department, which reports to government and stakeholders 
and maintains databases and informatics, protocol standard operating procedures, quality assessment and test improvement measures, long-term outcomes and cost effectiveness. These activities require that the screening program gives instruction, receives input and facilitates collaboration between local nursery personnel, coordinators who log and arrange workflows for samples and data, the screening laboratory, the follow-up testing laboratory, and medical experts in diagnosis, management, definitive therapy and long-term assessment.

Ongoing tracking of test performance metrics and outcomes must be part of every screening program, with regular communication and adjustments of all components as needed to improve sensitivity, specificity, turnaround times, follow-up care, cost effectiveness and outcomes. Sharing of information at every level makes the program efficient, but also affords opportunities for new insights. For example, because no lymphocyte subset data are typically collected at the same time as the DBS for TRECs, setting the TREC cutoff for follow-up in actual practice is not straightforward. However, if all infants with abnormal TREC results have a repeat TREC DBS made from the same liquid blood sample submitted for lymphocyte subset determination, precise correlations between TRECs and simultaneous total and naïve $\mathrm{T}$ cell subsets can be readily determined. In collaboration with immunology experts, appropriate cutoff levels for TRECs can thus be adjusted to meet the public health goal of efficiently providing timely intervention for infants with significant $\mathrm{T}$ cell insufficiency. Screening programs should reflect specific aspects of the local population and require immunologists to develop new understanding of aspects of screening, just as public health program leaders, traditionally not familiar with immune disorders, need to learn what immune tests must follow the TREC screen and which hospitals are qualified to perform HCT for newborns with SCID.

Screening programs yield incomparable information about disease incidence, spectrum and outcomes; but in contrast to typical academic clinical research with human subjects, screening has been deemed so critical to the public health interest that it can be conducted without individual informed consent. While state departments of public health have their own policies regarding who may decline screening, all states have had to make the determination that universal screening is required to identify individuals who may be affected and refer them to appropriate experts for care. Individual autonomy, confidentiality and voluntary consent can thus be viewed as inherently in conflict with the needs of the government and immunologists to diagnose and manage rare PIDs being screened for. Ongoing quality monitoring and new test development by screening programs require use of residual dried blood spot (DBS) samples that are archived stored. $(19,20)$ Addition of new diseases to newborn screening panels can be expected to reveal both anticipated information on disease incidence, spectrum and characteristics and unanticipated findings that challenge medical experts and engage basic scientists. Moreover, storage of residual DBS material collected for newborn screening opens the possibility of conducting much more far-reaching research, while with modern DNA sequencing technology one can sequence a person's entire genome using a small punch from a stored DBS. (21) Controversies and legal battles claiming breaches of the public trust have resulted in destruction of state biobanks of newborn DBS. Clearly, the lines between program monitoring and pure research are not always distinct. Important new information is generated from screening, strict rules for use of samples and data are needed to protect individuals, while allowing for essential functions 
of the screening program to improve patient care. Further investigational research should also be encouraged, but may require returning to the patient or family for informed consent unless links between individual identify and samples can be erased.

\section{Justification for Newborn Screening for SCID with the TREC Test}

The characteristics of SCID that merited its addition to panels of newborn screened disorders, as originally formulated by Wilson and Jungner (22) regarding screening for phenylketonuria (PKU), are its lack of recognizable features on physical exam, asymptomatic status in early life, high disease burden if untreated, an inexpensive screening test that is sensitive and specific, available effective treatment, improved survival and outcome for infants detected early. (23-26) What was initially missing was an appropriate high-throughput test. A simple CBC and differential to detect lymphopenia was suggested as a means to detect many cases of SCID, (24) but not all SCID genotypes with high B and NK cell numbers, SCID cases with maternal T cell engraftment, or leaky SCID cases with oligoclonal T cell expansion as seen in Omenn syndrome. $(23,25)$ The absolute lymphocyte count used as a cutoff would have to be set at a level that would result in unacceptably frequent false positive test results, and furthermore would require liquid blood samples, generally run at the point of care and problematic for screening programs to capture and standardize. Flow cytometry with liquid blood samples to determine $\mathrm{T}$ cell numbers was also proposed as a screening method, but the cost of obtaining and running a liquid blood sample for every infant rather than using the existing DBS sample to rule out problems in the large majority would be prohibitive. Protein assays for T cell specific markers such as CD3 chains were also tried, but had insufficient reproducibility due to the low amount of protein that could be recovered from DBS samples.

Another approach to SCID screening would be to detect DNA sequence variants in known SCID disease genes; these sequence variations could be either previously defined, or as-yet unreported mutations predicted to be deleterious. Increasingly, NBS laboratories that include cystic fibrosis in their screening test panel currently use DNA mutation arrays, with genomic sequencing as a second-tier test to detect further mutations. (27) However, gene sequencing is not currently suitable for primary screening for SCID because new pathogenic SCID mutations continue to be discovered. (28) and unique variants of uncertain significance are frequent.

The development of a diverse repertoire of T cells, each with its own T cell receptor (TCR), is essential for recognition of foreign antigen bound to self MHC molecules. To generate a large number of unique TCRs, DNA gene rearrangement and linear re-assembly occurs in thymocytes, with each cell having its own combination of a TCR elements. This recombination is mediated by enzymes that induce double strand breaks at specific sequences that flank each V, D and J segment. Following successful cutting and in-frame religation of the DNA, unique productively rearranged $\mathrm{T}$ cell receptors are generated, and $\mathrm{T}$ cell precursors expressing these receptors mature and undergo selection, eventually resulting in the diverse repertoire of naïve $\mathrm{T}$ cells released from the thymus into peripheral blood. Importantly, the excised DNA fragments of the TCR that are not destined to be incorporated into a recombinant receptor gene are also ligated at their ends, forming a variety of circular 
DNA byproducts called T cell receptor excision circles, or TRECs. $(2,29,30)$ One particular circular species, the $\delta$ rec- $\psi \mathrm{Ja}$ TREC, is produced late in maturation by $70 \%$ of all T cells that are destined to express $\alpha \beta$ TCRs. TRECs are stable, but are not replicated during mitosis. Therefore, they persist, but become diluted as T cells proliferate. TRECs can be readily detected by a PCR reaction using primers flanking the joint of the circle. Thus, the number of TREC copies is an indicator of thymic production of naïve $T$ cells.

The TREC assay was adapted to NBS as a real-time PCR method that amplifies DNA extracted from DBS to detect TRECs as a biomarker of naïve T cells. (23) Low or absent TRECs indicate insufficient naïve T cells, regardless of the underlying cause. A PCR control consisting of primers amplifying an unrelated genomic DNA segment (typically from the $\beta$ Actin or RNaseP gene) checks the quality of DNA in each DBS, distinguishing low TRECs due to genuinely low T cells vs. due to insufficient or poor-quality DNA. $(2,30)$ Thus the TREC test flags genetic causes of SCID or impaired T cell production as well as conditions in which there is increased loss of $\mathrm{T}$ cells from the peripheral circulation. The TREC assay became the first immune disorder for which NBS was possible, and at the same time became the first high-throughput DNA-based NBS test. (2) Introduction of DNA technology as a platform in all laboratories performing TREC detection has made additional DNA-based screening tests possible, including recently developed tests for spinal muscular atrophy (SMA). (31)

The TREC test was predicted by a cost analysis (32) and a Markov model (33) to be a costeffective strategy to save lives of infants with SCID under a range of assumptions about incidence and cost of early vs. late treatment for SCID. However, such hypothetical predictions can be replaced with actual experience now that programs are in place.

\section{Implementation of SCID NBS in the USA}

Following an evidence-based review and pilot TREC screening programs in Wisconsin, Massachusetts and the Navajo Reservation (where a founder mutation in the Artemis gene results in a frequency of SCID of one in 2,000 births), the Advisory Committee on Heritable Disorders in Newborns and Children made the recommendation, endorsed by the DHHS Secretary, that screening for SCID be added to the Recommended Uniform Screening Panel (RUSP) of newborn screened diseases in the USA. (34) States with the largest numbers of births, California, New York, Florida and Texas, as well as a number of other states, promptly added SCID screening to their NBS panels, so that by the end of 2013, over half of the births in the U.S. were being screened with a TREC test. In 2014, an analysis of early pooled data from 11 screening programs endorsed the effectiveness of TREC screening. (35) All laboratories followed general guidelines as published by the Clinical Laboratory Standards Institute, (36) but had their own assays, TREC cutoffs and rules for handling testing of ill and preterm infants in neonatal intensive care units (NICUs), reflecting each program's characteristics and population. Thus, there was variability in the criteria for recalling infants for additional specimens, implementation of referral to specialists for follow-up, and immunological investigations to evaluate infants with abnormal TREC results. (35) The 11-program report concluded that a) all programs readily detected infants with typical and leaky SCID; b) no SCID cases were recognized at that time to have been 
missed by NBS and then detected later (see update below); and c) affected infants were referred for immune restorative treatments in a timely fashion with a high rate of survival.

By November, 2018, 48 states plus Puerto Rico were screening all infants for SCID, as were an increasing number of nations and jurisdictions, including Israel, New Zealand, Norway, Taiwan, and several Canadian provinces. Additional regional or pilot programs are under way in Europe and the Middle East.

\section{Findings from SCID Newborn Screening in California}

\section{California SCID Population and Methods}

Lack of standardized practices has complicated analysis of aggregate performance data across programs, so available data from California, an ethnically diverse state with the highest number of births, are summarized here. (38) The experience of California alone is larger than the previously generated data from 11 pooled screening programs, and consistent follow-up practices have facilitated outcome tracking. Starting in August 2010, California screened newborns DBS for TRECs at the California Department of Public Health (CDPH) Genetic Disease Laboratory (GDL) using a laboratory-developed test, as published previously. (38) The EnLite neonatal TREC kit (PerkinElmer, Inc.) was adopted in March, 2016, after which testing was done in regional laboratories with oversight from the GDL. TREC and $\beta$-Actin copy number thresholds have been adjusted over time based on experience. As of 2017, infants with the equivalent of greater than 18 TRECs/ $\mu \mathrm{l}$ and adequate $\beta$-Actin with the EnLite assay have been considered not clinically immunodeficient and not tested further, while infants with fewer TRECs/ $\mu$ l on one or at most 2 DBS had follow-up lymphocyte subset measurement as part of the SCID NBS program. Liquid blood was sent to a single laboratory (Quest Diagnostics, Inc., San Juan Capistrano, CA) for complete blood counts and numbers and percentages of CD3+ T cells, CD19+ B cells, CD14+/CD56+/CD3natural killer (NK) cells, CD3+/CD4+ helper T cells, CD3+/CD8+ cytotoxic T cells, and CD45RA+ naïve-phenotype and CD45RO+ memory-phenotype helper and cytotoxic T cells, respectively. Lymphocyte phenotypes were interpreted by the immunology experts affiliated with the program.

Infants with fewer than $300 \mathrm{CD} 3 \mathrm{~T}$ cells/uL of blood or with less than $2 \%$ of helper $\mathrm{T}$ cells that were naïve $(C D 45 R A+)$ were referred for immediate admission to pediatric hospitals with experience in treating SCID (Children's Hospital of Los Angeles, Stanford Lucille Packard Children's Hospital, University of California Los Angeles Mattel Children's Hospital, or University of California San Francisco Benioff Children's Hospital); while those with up to 1,500 circulating $\mathrm{T}$ cells $/ \mu \mathrm{l}$ and greater than $2 \%$ naïve $\mathrm{T}$ helper cells, defined as non-SCID T cell lymphopenia (TCL), were referred to pediatric immunology specialty clinics at these and additional sites for diagnosis and management. The definitions were chosen to be highly selective, but to avoid exposing infants with significant immune compromise to the otherwise-mandated live, attenuated rotavirus vaccine, which protects healthy infants, but can cause serious diarrhea in those with immunodeficiency. (39)

In the 6.5 years between August 15, 2010, and March 31, 2017, California screened $3,252,156$ newborns for SCID (Figure 2). (37) Of these, only 562 (1/5,800 total births, or 
less than $0.2 \%$ of births) had non-normal TREC results requiring liquid blood lymphocyte phenotyping. Although only $9 \%$ of all infants were cared for in NICUs, these infants were $53 \%$ of the 562 who needed liquid follow-up blood samples (Figure 2, right, grey shading).

TCL was documented in 38\% of infants requiring follow-up flow cytometry $(n=213)$, or one per 15,300 total newborns, of whom 50 had SCID and the rest had non-SCID TCL conditions (Figure 2). An urgent positive result (0-3 TRECs/ $\mu$ ) had a high positive predictive value for SCID and non-SCID TCL; of the 82 newborns with urgent positive TREC results, 38 (46\%) had SCID and 21 (26\%) had non-SCID TCL. Ethnicity and sex were not significant risk factors for having an abnormal TREC screen or low $\mathrm{T}$ cell number. Follow-up for 1-7 years was solicited from transplant centers and immunologists.

\section{SCID found by NBS}

Of the 50 infants with an immuno-phenotype of SCID, the genotype distribution is shown in Figure 3. One infant born to a foreign visitor left the USA before receiving any further care. The others were healthy at diagnosis at a mean of 21 days of age (range 0-40 days), except for two who had sought medical attention for infection or rashes and one preterm infant with feeding intolerance, bronchiolitis and leukopenia. (40) Only two infants identified by SCID NBS were known by their doctors to be at risk for SCID due to a positive family history, while seven others in retrospect had older affected relatives with SCID, and one had a sibling who had died of infantile pneumonia consistent with SCID.

Of the 49 evaluable patients who were treated 46 survived (94\%) after receiving allogeneic HCT, autologous corrected cell GT, or ERT (Table 1). Of the three deaths, one infant of unknown genotype died of cytomegalovirus (CMV) infection. One with RAG1-deficient SCID and one with X-linked SCID, each of whom had received pre-transplant conditioning with high dose busulfan chemotherapy, developed fatal hepatic sinusoidal obstruction syndrome post-HCT; one of these infants also had CMV infection that had been present prior to HCT. While about $90 \%$ of cases with typical SCID had urgent positive TREC results, half of cases with leaky SCID had TREC numbers in the positive, but not urgent range (data not shown). The right columns of Table 1 summarize outcomes after therapy. As reported previously, the X-linked $I L 2 R G$ gene was mutated most frequently, but accounted for only $28 \%$ of cases detected by NBS, in contrast to nearly half of the cases from series published prior to newborn screening. (1) Also, as has been recognized for this genotype and JAK3 deficiency (both defects affecting the same interleukin-2, -4, -7, $-9,-15$ and -21 cytokine receptor pathways), unconditioned haploidentical transplants (MMRD, Table 1) reconstituted $\mathrm{T}$ cell, but not B cell immunity, causing continued dependence on IgG infusions in six infants with X-linked IL2RG and one with JAK3 mutations. $(1,2,6,7)$ Infants with these genotypes who received conditioned allogeneic transplants or GT with low-dose busulfan were more likely to have B cell reconstitution enabling immunoglobulin (IgG) infusions to be discontinued. The nine infants with ADA-deficient SCID had, in addition to T, B, and NK lymphopenia, fewer neutrophils than healthy infants (mean 986 vs. 4,700/ $\mu \mathrm{l}$ for ADA deficient vs. healthy infants).

GT, consisting of autologous transplantation of bone marrow derived hematopoietic stem cells transduced with a vector encoding the correct cDNA, was used to treat four infants with 
$\mathrm{X}$-linked and eight with ADA-deficient SCID, all of whom are alive 2.5-6.5 years after treatment. All infants receiving GT for ADA-deficient SCID have had multi-lineage immune reconstitution and no longer require IgG infusions. Three recipients of GT for X-linked SCID have also recovered T-cell immunity, one of whom had failed his initial haploidentical T-cell-depleted allogeneic HCT; one of these patients no longer requires IgG infusions. However, one recipient of GT for X-linked SCID failed this initial treatment, but recovered $\mathrm{T}$ - and B-cell immunity after an allogeneic transplant from a matched unrelated donor.

Deficiency of RAG1 or RAG2 caused four typical and seven leaky cases of SCID, three of the latter having Omenn syndrome, in which oligoclonal, dysregulated T cells expand and cause rashes, adenopathy, splenomegaly, eosinophilia and elevated IgE. An example was an infant with leaky RAG1 SCID who had over 4,500 T cells/uL. Although this was in the normal range, only 46 of his 3,680 CD4 helper T cells (1.2\%) had the naïve CD45RA phenotype.

Despite clinical sequencing of known SCID genes, five infants with SCID (10\%) remained without a proven genotype. Whole-exome sequencing failed to solve four of these, while confirmatory experiments revealed one infant to have a defect in a novel SCID gene,

BCL11B. (42)

\section{Missed cases of leaky SCID that presented after the newborn period}

Two cases of SCID were discovered to have been missed by NBS. One infant, who presented at age seven months with Pneumocystis pneumonia, had the known, recurrent, hypomorphic X-linked IL2RG mutation Arg222Cys. (42) His delayed-onset, leaky SCID was successfully treated with a conditioned HCT from a matched unrelated donor. The other was a delayed-onset case of incomplete ADA-deficiency, diagnosed at 23 months of age after repeated otitis and hospitalization for pneumonia. (43) This child was successfully treated with GT. Both infants had newborn DBS TREC numbers well above the cutoff for follow-up testing. Their mutations were sufficiently leaky to allow some $\mathrm{T}$ cell maturation with normal TREC production during the neonatal period, but by the time of diagnosis $\mathrm{T}$ cell insufficiency had led to infections as well as low T cell numbers and TRECs. Importantly, raising the TREC cutoff in the California SCID screening program would not have helped to identify either of these cases by NBS. They illustrate that SCID, like most single gene disorders, has a broad spectrum of mutations and phenotypic presentations, that rarely (in fewer than one in a million births in the California experience) can have false negative both TREC NBS.

\section{Non-SCID T-Cell Lymphopenia}

Infants with non-SCID TCL were classified as listed in Table 2 and detailed in Figure 4. While most of these 72 cases received a definitive diagnosis in the neonatal period, 22 required further testing over three to 18 months to establish a diagnosis (Figure 4, grey shading). The most frequent non-SCID TCL conditions were congenital syndromes (Figure 4, Table 2). Six additional infants with low TRECs and syndromes died before flow cytometry could be performed (not shown). Two thirds of all syndromic cases of non-SCID TCL had DiGeorge syndrome (Table 2). Over 90\% of these had partial DiGeorge syndrome, 
in which $\mathrm{T}$ cell numbers rose over time and $\mathrm{T}$ cell function was judged by their immunology providers to be adequate. However, four infants with complete DiGeorge syndrome, having persistently fewer than $300 \mathrm{~T}$ cells/ $\mu \mathrm{l}$ and/or absent naïve helper $\mathrm{T}$ cells, received thymus transplantation therapy in an experimental protocol at Duke University, Durham, NC, three of whom have survived for at least two years. (44-46) Trisomy 21 accounted for $8(11 \%)$ infants with syndromes; TCL resolved in three other cases of partial DiGeorge syndrome, three died of non-immune complications, and two were lost to follow-up. All five ataxiatelangiectasia (AT) patients were apparently healthy, term newborns from regular nurseries with $800-1,323 \mathrm{~T}$ cells/ $\mu$ l. Rising alpha-fetoprotein levels after age six months or sequencing panels of genes or exomes led to identification of homozygous or compound heterozygous deleterious mutations in the ATM gene. (47) One infant with AT developed infections requiring IgG infusions during his first year. One infant with CHARGE syndrome was considered for thymus transplantation, but died of respiratory failure. Single patients with very rare conditions constituted the remainder of cases with syndromes, including one with a novel short-limbed immuno-skeletal dysplasia in whom whole exome sequencing revealed EXTL3 deficiency (48) and one with $R A C 2$ mutation as described in a single previous case. (49)

Secondary TCL in 25 cases (Figure 4) resolved to normal once excess T-cell losses or suppression of T-cell maturation had been reversed. T-cell losses were associated with vascular leakage, chylothorax, and intestinal atresia or gastroschisis; while two infants had T-cell suppression due to in utero exposure to maternal medications, fingolimod and azathioprine, respectively, and one infant had a thymic teratoma, with $\mathrm{T}$ cells normalizing after it was resected. Self-resolving TCL was also associated with preterm birth, generally affecting infants born at or before 32 weeks' gestation.

Idiopathic TCL included cases for which an underlying condition could not be determined, even after immunologic evaluation and in many instances sequencing of gene panels or whole exomes or genomes. Of the 33 cases with idiopathic TCL 13 resolved or improved while 13 were persistent and six were lost to follow-up.

\section{Lessons from SCID Newborn Screening}

\section{Anticipated outcomes}

TREC screening for SCID has established the feasibility and utility of DNA-based, highthroughput screening, which, like tandem mass spectrometry, has already proven adaptable to additional conditions, including spinal muscular atrophy as approved in July, 2018, for addition to the Recommended Uniform Screening Panel. (31) As expected, TRECs have now been shown to be a highly specific and sensitive biomarker for the phenotype of $\mathrm{T}$ cell insufficiency, regardless of underlying genotype. Therefore, the California program based on screening newborns with the TREC test to identify and diagnose SCID has facilitated identification in pre-symptomatic infants with known and unknown SCID defects, permitting timely treatment with excellent survival. Definitive diagnosis of SCID has had to rely on laboratory test criteria (50) instead of formerly used clinical features related to infectious complications, such as failure to thrive; diarrhea; and recurrent, serious and opportunistic infections. Lentiviral gene therapy has become increasingly available when the 
genotype is known, and when administered with low dose busulfan can achieve multilineage transduction and fully restore adaptive immunity in SCID caused by mutations in the $A D A$ gene and the X-linked $I L 2 R G$ gene. A similar protocol for DCLRE1C (or Artemis) mutant SCID is now open at UCSF and appears promising as well. (51) Based on our observed genotype frequencies for SCID (Figure 3), around half of all infants with SCID may be eligible for gene therapy in the near future. Experimental thymus transplantation has also been successful in selected cases of DiGeorge thymic insufficiency, but is not widely available. (44-46)

Integrating lymphocyte phenotyping by flow cytometry as a follow-up test within the SCID NBS program in California has proven efficient and cost-effective. Performance in a single contract laboratory has minimized cost and assured that the screening program captures results for outcome tracking, adjustment of the cutoff for the TREC screening test, and quality assurance, while allowing nearly $60 \%$ of the cases with abnormal TREC results to be dismissed without a costly, time-consuming evaluation by an immunology specialist (Figure 2). Clinically significant non-SCID TCL, defined as fewer than $1,500 \mathrm{~T}$ cells $/ \mu \mathrm{l}$, has been recognized as a rare phenomenon (one per 20,000 births); identifying this small group of infants so that they can avoid possible harm from receiving live attenuated rotavirus vaccine allows all other infants to be vaccinated, assuring maximum effectiveness of vaccination as a public health measure.

Given its high degree of specificity, the TREC test for SCID has not generated excessive anxiety on the part of families or negative feedback from primary providers.

Communications between screening program staff and immunologists, as well as follow-up lymphocyte phenotyping within the NBS program have assured that infants with positive tests have a prompt, consistent approach to definitive diagnosis. Persistent idiopathic TCL is infrequent in our program, with an incidence of one per 100,000 when a T cell subset determination is incorporated into newborn screening and syndromic diagnoses are made promptly.

\section{Unanticipated findings}

Population based, unbiased NBS with TRECs has also revealed findings that were not anticipated. The overall incidence of SCID, 1/65,000 births (95\%CI 1/51,000-1/90,000) in California, is higher than was estimated prior to screening. (4,52) Family history of SCID has not been as helpful as one would hope; existence of prior affected relatives was often not recognized by the care team prenatally, but rather solicited only in retrospect after identification of SCID through screening. In contrast to case series published prior to screening, SCID genotypes with NBS revealed higher proportions of autosomal recessive and leaky SCID cases, perhaps reflecting the higher likelihood for such cases to have no positive family history or to present to medical attention later in infancy or childhood. Although exceptionally rare, missed cases of leaky, late-onset SCID have now appeared, necessitating vigilance on the part of pediatricians and immunologists to consider T cell deficiencies in children presenting with frequent or opportunistic infections.

Discovery of TCL at birth in ataxia telangiectasia was a surprise, as without NBS this disease was formerly diagnosed only after the appearance of neurologic manifestations, 
ocular telangiectasias or frequent infections in childhood. (47) Trisomy 21, CLOVES syndrome, and RAC2 deficiency were not associated with newborn TCL prior to NBS for SCID.

Similarly, the original concept was that newborn screening could eliminate infectious complications of SCID by having infants recognized prior to the natural waning of maternal $\operatorname{IgG}$ antibodies that are transferred to the fetus starting at about 33 weeks of gestation. However, CMV has been a serious and even fatal infection in newborn screened SCID infants. Nosocomial infections have occurred, and herpesviruses other than CMV as well as adenoviruses are known to be highly pathogenic in the absence of T-cell immunity. Exposures to most infections can be reduced with isolation, handwashing and administration of IgG and prophylactic antimicrobials to infants with SCID diagnosed by NBS. But these measures and even shortening TREC test turnaround times to a few days cannot prevent exposure to CMV through vaginal secretions or breastmilk if the mother has had prior infection. (53) Further study will be required to establish the sources and best preventive measures to combat CMV and other infections.

Finally, population-based screening for insufficient $\mathrm{T}$ cells provides the opportunity to discover new genetic defects of $\mathrm{T}$ cell immunity, revealing information about $\mathrm{T}$ cell developmental pathways that are important in humans. Mutations in $B C L 11 B(41)$ and EXTL3 (48) were newly discovered T cell deficiency diseases following NBS in California; infants detected by screening who lacked defects in known SCID genes were enrolled in research protocols to undergo exome sequencing and functional analysis of variants. Population-based detection of SCID and non-SCID TCL by TREC screening will undoubtedly continue to reveal infants with new genes and immuno-developmental pathways that are essential for human $\mathrm{T}$ cell development.

\section{Conclusion}

The combination of relative ease of implementation and importance supports the rapid adoption of TREC screening for SCID that has occurred in the USA and, increasingly, worldwide. TREC testing brings into NSB laboratories high-throughput, DNA-based methodology applicable to screens for other conditions, such as spinal muscular atrophy, as well as deep sequencing for diagnostic level testing rather than just screening. As an early adopter of TREC NBS, the California NBS program for SCID has demonstrated detection of SCID and clinically important non-SCID TCL with near-complete sensitivity and outstanding specificity, strengthening the live rotavirus vaccination program by identifying the infants with immune compromise who should not receive it. The incidence of SCID has proven higher than originally thought, and new disease genes have been discovered in infants identified by SCID NBS. Treatment of SCID detected by NBS using allogeneic HCT and autologous gene therapy has led to $94 \%$ survival with a high rate of full immune reconstitution. Areas of focus for further improvement are in targeted chemotherapy dosing and avoidance of CMV and other infections. 


\section{Acknowledgements}

Thanks to Fred Lorey, Bob Currier. George Amatuni and the diligent team at the California Department of Public Health Newborn Screening Program for their help with data analysis and their efforts on behalf of newborn infants. Dr. Puck received grants from U54 AI 082973 for the Primary Immune Deficiency Treatment Consortium, a member of the Rare Diseases Clinical Research Network (RDCRN) funded by NIAID and ORDR, NCATS; and NIH; R01 AI105776, NIAID, NIH. Support was also provided by the SCID families, the Jeffrey Modell Foundation, the SCID Angels for Life, the Immune Deficiency Foundation, the Lisa and Douglas Goldman Fund and the Michelle Platt-Ross Foundation.

\section{References}

1. Buckley RH. Molecular defects in human severe combined immunodeficiency and approaches to immune reconstitution. Annu Rev Immunol 2004;22:625-655. [PubMed: 15032591]

2. Kwan A, Puck JM. History and current status of newborn screening for severe combined immunodeficiency. Sem Perinatol 2015;39:194-205.

3. Gatti RA, Meuwissen HJ, Allen HD, Hong R, Good RA. Immunological reconstitution of sex-linked lymphopenic immunological deficiency. Lancet 1968;817;2(7564): 388-389. [PubMed: 4173794]

4. Buckley RH, Schiff SE, Schiff RI, et al. Hematopoietic stem-cell transplantation for the treatment of severe combined immunodeficiency. N Engl J Med 1999;340:508-516. [PubMed: 10021471]

5. Dvorak CC, Cowan MJ, Logan BR, et al. The natural history of children with severe combined immunodeficiency: baseline features of the first fifty patients of the Primary Immune Deficiency Treatment Consortium prospective study 6901. J Clin Immunol 2013;33:1156-1164. [PubMed: 23818196]

6. Pai SY, Logan BR, Griffith LM, et al. Transplantation outcomes for severe combined immunodeficiency, 2000-2009. New Engl J Med 2014;371:434-446. [PubMed: 25075835]

7. Heimall J, Logan BR, Cowan MJ, et al. Immune Reconstitution and survival of 100 SCID patients post hematopoietic cell transplant: A PIDTC natural history study. Blood 2017;130:2718-2727. [PubMed: 29021228]

8. Myers LA, Patel DD, Puck JM, Buckley RH Hematopoietic stem cell transplantation for SCID in the neonatal period leads to superior thymic output and improved survival. Blood 2002;99:872-878. [PubMed: 11806989]

9. Chan A, Scalchunes C, Boyle M, Puck JM. Early vs. delayed diagnosis of severe combined immunodeficiency: A family perspective survey. Clin Immunol 2011;138:3-8. [PubMed: 21035402]

10. Picard C, Bobby Gaspar H, Al-Herz W, et al. International Union of Immunological Societies: 2017 Primary Immunodeficiency Diseases Committee Report on Inborn Errors of Immunity. J Clin Immunol 2018;38:96-128. [PubMed: 29226302]

11. Landrum MJ, Lee JM, Benson M, et al. ClinVar: improving access to variant interpretations and supporting evidence. Nucleic Acids Res 2018;46(D1): D1062-D1067. [PubMed: 29165669]

12. Bridges RA, Berendes H, Good RA.. A fatal granulomatous disease of childhood; the clinical, pathological, and laboratory features of a new syndrome. AMA J Dis Child 1959; 97:387-408. [PubMed: 13636694]

13. Holland SM. Chronic granulomatous disease. Hematol Oncol Clin North Am 2013; 27:89-99. [PubMed: 23351990]

14. Shaw KL, Garabedian E, Mishra S, et al. Clinical efficacy of gene-modified stem cells in adenosine deaminase-deficient immunodeficiency. J Clin Invest 2017;127:1689-1699. [PubMed: 28346229]

15. De Ravin SS, Wu X, Moir S, et al. Lentiviral hematopoietic stem cell gene therapy for X-linked severe combined immunodeficiency. Sci Transl Med 2016;8(335):335ra57.

16. Mamcarz E, Zhou S, Lockey T, et al. Interim results from a phase I/II clinical gene therapy study for newly diagnosed infants with X-linked severe combined immunodeficiency using a safetymodified lentiviral vector and targeted reduced exposure to busulfan. Blood 2017 130:523, Oral Abstract 801.

17. Kohn DB, Hershfield MS, Puck JM, et al. Consensus approach for the management of severe combined immune deficiency caused by adenosine deaminase deficiency. $\mathrm{J}$ Allergy Clin Immunol 2018;S0091-6749(18):31268-5. 
18. Kohn DB, Kuo CY. New frontiers in the therapy of primary immunodeficiency: From gene addition to gene editing. J Allergy Clin Immunol 2017;139:726-732. [PubMed: 28270364]

19. Botkin JR, Goldenberg AJ, Rothwell E, Anderson RA, Lewis MH. Retention and research use of residual newborn screening bloodspots. Pediatrics 2013;131:120-127. [PubMed: 23209103]

20. Feuchtbaum L, Yang J, Currier R. Follow-up status during the first 5 years of life for metabolic disorders on the federal Recommended Uniform Screening Panel. Genomics in Med 2018;20:831839.

21. Bassaganyas L, Freedman G, Vaka D, et al. Whole exome and whole genome sequencing with dried blood spot DNA without whole genome amplification. Hum Mutat 2018;39:167-171. [PubMed: 29067733]

22. Wilson JM; Jungner YG (1968). "Principles and practice of mass screening for disease". Boletin de la Oficina Sanitaria Panamericana. Pan American Sanitary Bureau. 65 (4): 281-393.

23. Chan K, Puck JM. Development of population-based newborn screening for severe combined immunodeficiency. J Allergy Clin Immunol. 2005;115:391-398. [PubMed: 15696101]

24. Buckley RH. The long quest for neonatal screening for severe combined immunodeficiency. $\mathbf{J}$ Allergy Clin Immunol 2012;129:597-604. [PubMed: 22277203]

25. Puck JM: Laboratory technology for population-based screening for severe combined immunodeficiency in neonates: the winner is T-cell receptor excision circles. J Allergy Clin Immunol 2012;129:607-616. [PubMed: 22285280]

26. Verbsky JW, Baker MW, Grossman WJ, et al. Newborn screening for severe combined immunodeficiency; the Wisconsin experience (2008-2011). J Clin Immunol 2012;32:82-88. [PubMed: 22068910]

27. Kharrazi M, Yang J, Bishop T, et al. Pediatrics. Newborn Screening for Cystic Fibrosis in California. 2015;136:1062-1072.

28. Lebet T, Chiles R, Hsu AP, Mansfield E, Warrington JA, Puck JM. Mutations causing severe combined immunodeficiency: detection with a custom resequencing microarray. Genet Med 2008;10:575-585. [PubMed: 18641513]

29. Douek DC, McFarland RD, Keiser PH, et al. Changes in thymic function with age and during the treatment of HIV infection. Nature 1998;396:690-695. [PubMed: 9872319]

30. Hazenberg MD, Otto SA, Cohen SJW, et al. Increased cell division but not thymic dysfunction rapidly affects the T-cell receptor excision circle content of the naive T cell population in HIV-1 infection. Nat Med 2000;6:1036-1042. [PubMed: 10973325]

31. https://www.hrsa.gov/sites/default/files/hrsa/advisory-committees/heritable-disorders/reportsrecommendations/sma-final-report.pdf.SMAFinalReport-hrsa.gov, accessed November 6, 2018.

32. McGhee SA, Stiehm ER, McCabe ER. Potential costs and benefits of newborn screening for severe combined immunodeficiency. J Pediatr 2005;147:603-608. [PubMed: 16291349]

33. Chan K, Davis J, Pai SY, Bonilla FA, Puck JM, Apkon M. A Markov model to analyze costeffectiveness of screening for severe combined immunodeficiency (SCID). Mol Genet Metab 2011;104:383-389. [PubMed: 21810544]

34. https://www.hrsa.gov/sites/default/files/hrsa/advisory-committees/heritable-disorders/rusp/ previous-nominations/scid-27-june-2018.pdf. Newborn screening for severe combined immunodeficiency: A summary of the evidence and Advisory Committee decision. Report date 29 April, 2009. Accessed November 7, 2018.

35. Kwan A, Abraham RS, Currier R, et al. Newborn screening for severe combined immunodeficiency in 11 screening programs in the United States. JAMA 2014;312:729-738. [PubMed: 25138334]

36. Hannon WH, Abraham RS, Kobryinski L, et al. Newborn blood spot screening for severe combined immunodeficiency by measurement of T-cell receptor excision circles; Approved guideline. Wayne, PA: Clinical and Laboratory Standards Institute, 2013 CLSI document NBS06A.

37. Amatuni G, Currier R, Church JA, et al. Newborn Screening for Severe Combined Immunodeficiency and T-cell Lymphopenia in California. Pediatrics 2018, In Press. 
38. Kwan A, Church JA, Cowan MJ, et al. Newborn screening for severe combined immunodeficiency and T-cell lymphopenia in California: Results of the first two years. J Allergy Clin Immunol 2013;132:140-150. [PubMed: 23810098]

39. Bakare N, Menschik D, Tiernan R, et al., Severe combined immunodeficiency (SCID) and rotavirus vaccination: reports to the Vaccine Adverse Events Reporting System (VAERS), Vaccine 2010;28(40):6609-6612. [PubMed: 20674876]

40. Buchbinder D, Puthenveetil G, Soni A, et al. Newborn screening for severe combined immunodeficiency: an opportunity for intervention. J Perinatology 2013;33:657-658.

41. Punwani D, Zhang Y, Yu J, et al. Multisystem Anomalies in Severe Combined Immunodeficiency with Mutant BCL11B. N Engl J Med 2016;375:2165-2176. [PubMed: 27959755]

42. Fuchs $\mathrm{S}$, Rensing-Ehl A, Erlacher M, et al. Patients with $\mathrm{T}^{+} /$low $\mathrm{NK}^{+}$IL-2 receptor $\gamma$ chain deficiency have differentially-impaired cytokine signaling resulting in severe combined immunodeficiency. Eur J Immunol 2014;44:3129-3140. [PubMed: 25042067]

43. la Marca G, Canessa C, Giocaliere E, et al. Tandem mass spectrometry, but not T-cell receptor excision circle analysis, identifies newborns with late-onset adenosine deaminase deficiency. $\mathrm{J}$ Allergy Clin Immunol 2013;131:1604-1610. [PubMed: 23280131]

44. Markert ML, Devlin BH, McCarthy EA. Thymus transplantation. Clin Immunol 2010;135:236246. [PubMed: 20236866]

45. Lee JH, Markert ML, Hornik CP, McCarthy EA, Gupton SE, Cheifetz IM, Turner DA. Clinical course and outcome predictors of critically ill infants with complete DiGeorge anomaly following thymus transplantation. Pediatr Crit Care Med 2014;15:e321-e326. [PubMed: 25068252]

46. Davies EG, Cheung M, Gilmour K, et al. Thymus transplantation for complete DiGeorge syndrome: European experience. J Allergy Clin Immunol 2017;140:1660-1670. [PubMed: 28400115]

47. Mallott J, Kwan A, Church J, Gonzalez-Espinosa D, Lorey F, Tang LF, Sunderam U, Sadhna R, Srinivasan R, Brenner SE, Puck JM. Newborn screening for SCID identifies patients with ataxia telangiectasia. J Clin Immunol, 33(3):540-549, 4 2013 PMCID: PMC3591536. [PubMed: 23264026]

48. Volpi S, Yamazaki Y, Brauer PM, et al. EXTL3 mutations cause skeletal dysplasia, immune deficiency, and developmental delay. J Exp Med. 2017;214:623-637. [PubMed: 28148688]

49. Accetta D, Syverson G, Bonacci B, et al. Human phagocyte defect caused by a Rac2 mutation detected by means of neonatal screening for T-cell lymphopenia. J Allergy Clin Immunol 2011;127:535-538.e1-2. [PubMed: 21167572]

50. Shearer WT, Dunn E, Notarangelo LD, et al. Establishing diagnostic criteria for severe combined immunodeficiency disease (SCID), leaky SCID, and Omenn syndrome: the Primary Immune Deficiency Treatment Consortium experience. J Allergy Clin Immunol 2014;133:1092-1098. [PubMed: 24290292]

51. Punwani D, Kawahara M, Yu J, et al. Lentivirus mediated correction of Artemis-deficient severe combined immunodeficiency. Hum Gene Therapy 2016;28:112-124.

52. Stephan JL, Vlekova V, Le Deist F, et al. Severe combined immunodeficiency: a retrospective single-center study of clinical presentation and outcome in 117 patients. J Pediatr 1993;123:564572. [PubMed: 8410508]

53. Hamprecht K, Maschmann J, Vochem M, Dietz K, Speer CP, Jahn G. Epidemiology of transmission of cytomegalovirus from mother to preterm infant by breastfeeding. Lancet. 2001;357(9255):513-518. [PubMed: 11229670] 
Clinical $\Rightarrow$ Laboratory $\Rightarrow$ Gene $\Rightarrow$ Genotype/Phenotype Observation Definition Diagnosis Spectrum

Basic knowledge, understanding, disease models

\begin{tabular}{|c|c|c|}
\hline $\begin{array}{l}\text { Treatment of } \\
\text { Complications } \\
\text { Antibiotics } \\
\text { Steroids }\end{array}$ & $\begin{array}{l}\text { Generic } \\
\text { Approaches to } \\
\text { Therapy } \\
\text { Immunoglobulin } \\
\text { Hematoipoietic } \\
\text { cell transplant }\end{array}$ & $\begin{array}{l}\text { Gene Specific } \\
\text { Therapies } \\
\text { Targeted Biologics } \\
\text { Gene Therapy } \\
\text { Gene Editing }\end{array}$ \\
\hline & & $\begin{array}{l}\text { Population Screening } \\
\text { TREC Newborn } \\
\text { Screening for SCID }\end{array}$ \\
\hline
\end{tabular}

Figure 1.

Evolution of knowledge and patient care in primary immunodeficieny. 


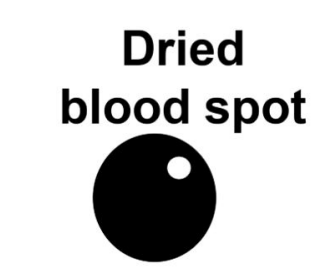

Liquid blood sample

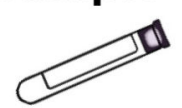

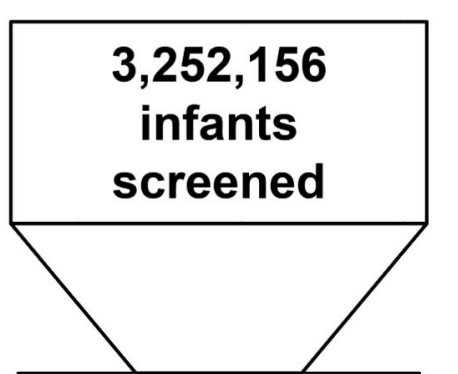

562 required flow cytometry

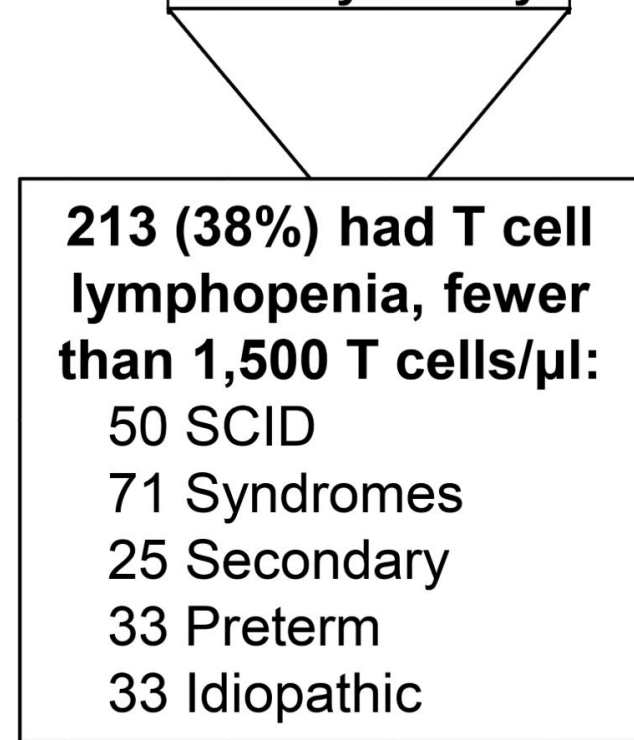

29
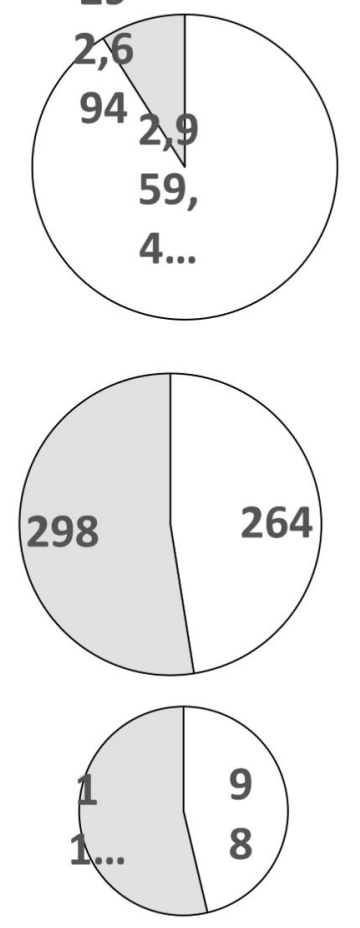

$\square$ Regular nursery

$\square$ NICU

Figure 2.

Newborns screened for SCID in California showing number of infants at each screening stage (left) and proportion in neonatal intensive care unit (NICU) (right). Total infants screened included $91 \%$ cared for in a regular well-infant nursery, and $9 \%$ in a neonatal intensive care unit (NICU). Infants requiring a liquid blood sample tested after one positive or 2 incomplete TREC tests (53\% NICU) were $0.17 \%$ of all births. Infants with fewer than $1,500 \mathrm{~T}$ cells $/ \mu \mathrm{L}$ or $<2 \%$ naïve CD3 CD4 CD45RA T helper cells (54\% NICU) were $0.007 \%$ of all births (1 per 15,000). 


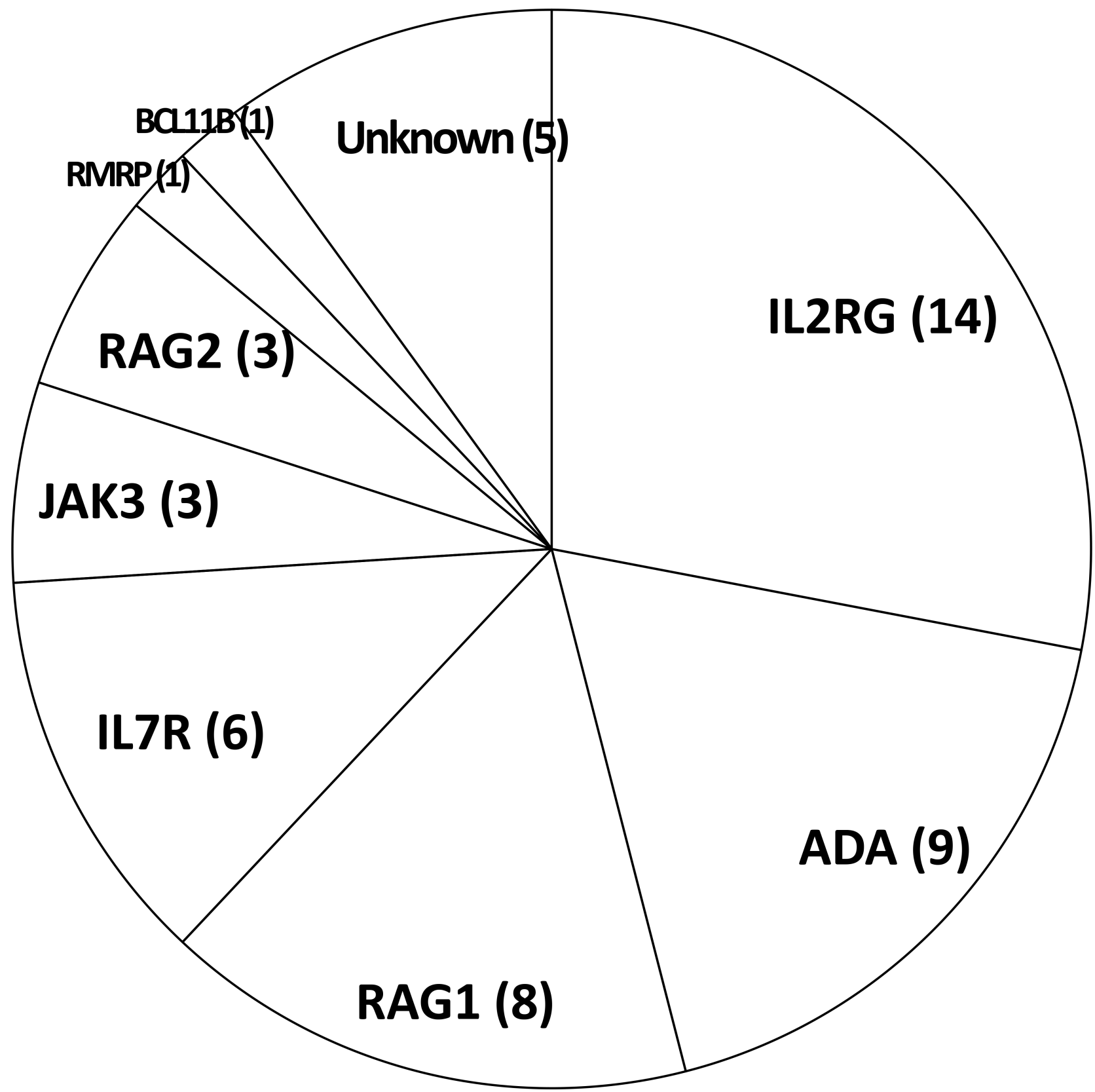

Figure 3.

Genotypes of 50 SCID cases found by newborn screening in California, 2010-2017. 


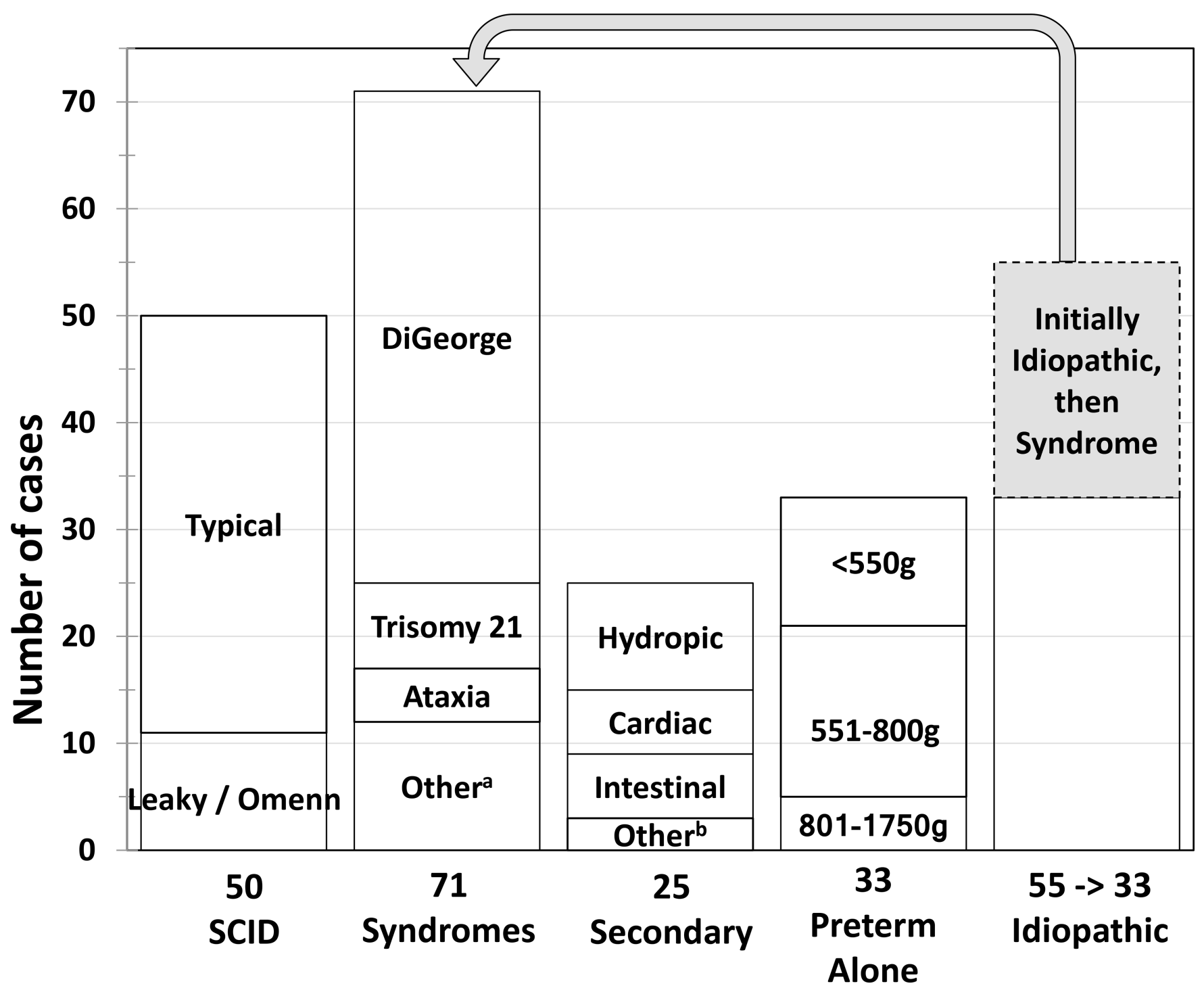

Figure 4.

Diagnoses of infants with T cell lymphopenia (TCL) detected by SCID newborn screening, including 50 with SCID (adapted from ref $\mathrm{xx}$ ), 71 with a syndrome in which $\mathrm{T}$ cells may be insufficient, 25 with TCL secondary to other neonatal conditions, 33 with preterm birth alone, and 33 idiopathic. Forty percent of the 55 initially idiopathic cases were confirmed to have a syndrome after up to 1 year of follow-up. Of the remaining 33 infants 13 resolved or improved spontaneously over time.

a For details see table 2.

b Includes in utero exposure to maternal immunosuppressive medications ( 1 instance each of fingolimod, azathioprine), teratoma of the thymus 


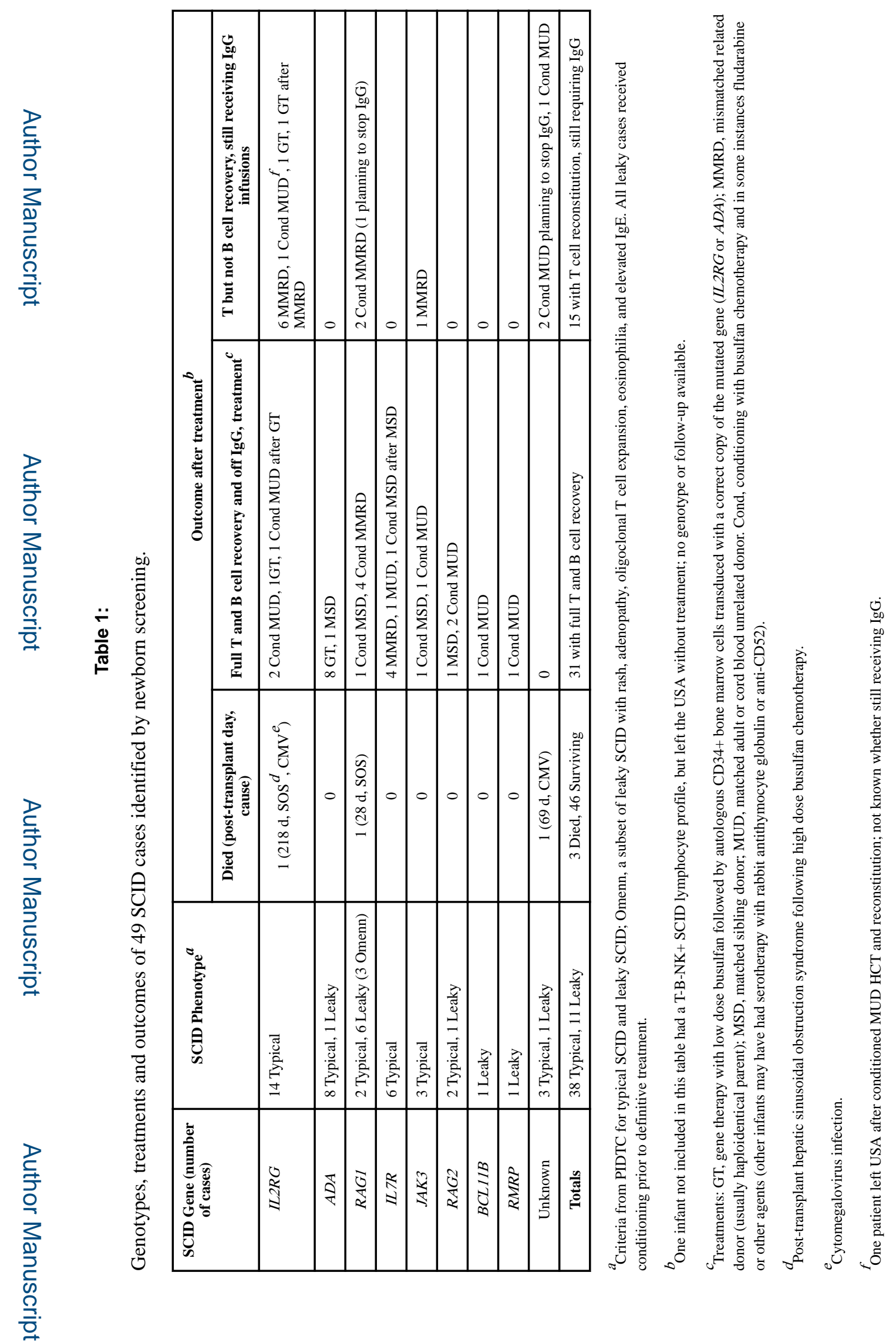

Immunol Rev. Author manuscript; available in PMC 2020 January 01. 


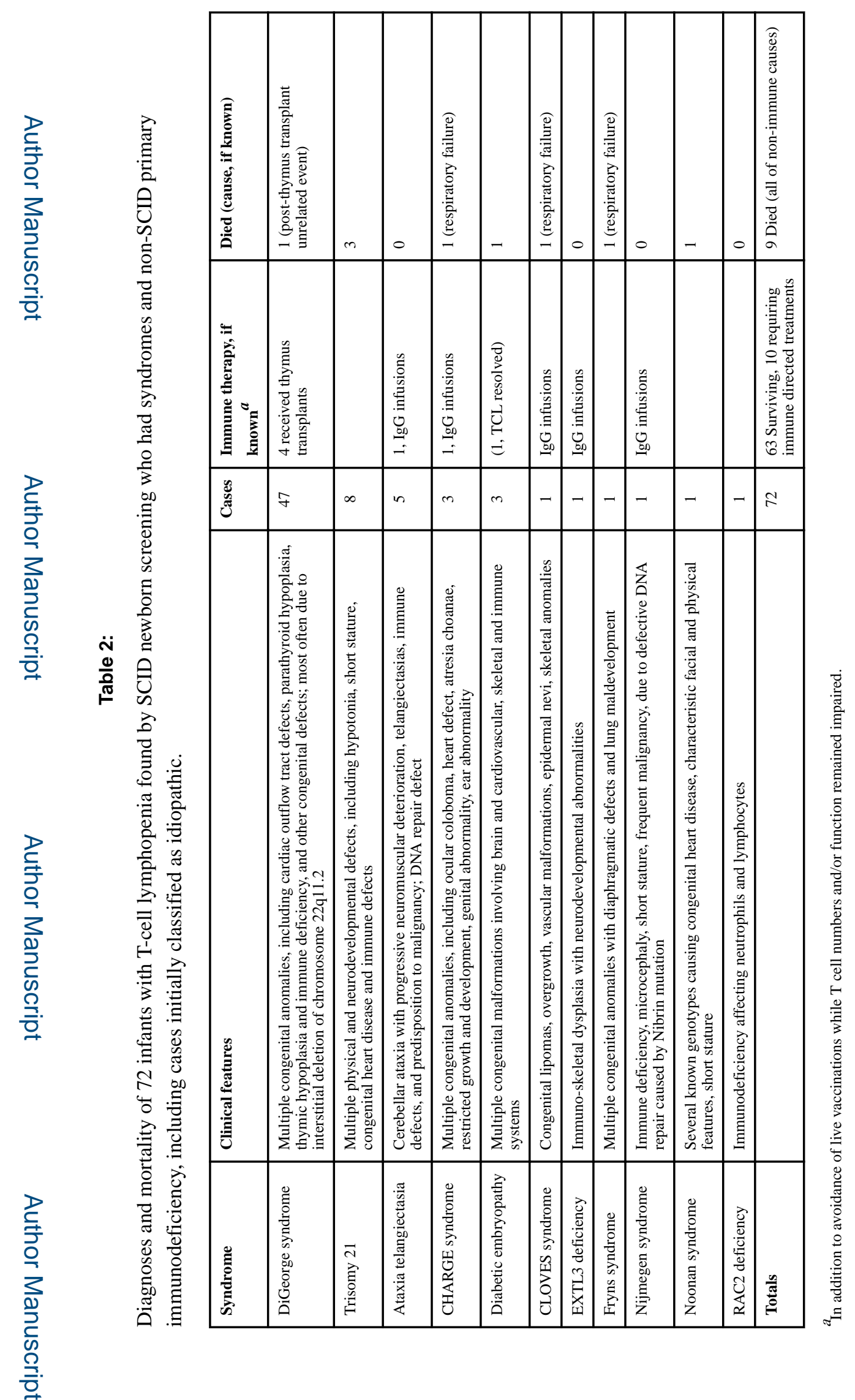

Immunol Rev. Author manuscript; available in PMC 2020 January 01. 